\title{
Claves educativas para aumentar la motivación de los jóvenes físicamente inactivos
}

\author{
Eva Sanz, $\mathrm{M}^{\mathrm{a}}$. Ángeles Valdemoros, Rafael Ramos y Ana Ponce de León \\ Universidad de La Rioja (España)
}

El presente estudio pretende averiguar, por una parte, cuáles son las razones que llevan a los jóvenes a abandonar la práctica físico-deportiva y, por otra, descubrir por qué algunos jóvenes nunca se iniciaron en este tipo de experiencias, con el fin de establecer una filosofía educativa que envuelva la práctica físico-deportiva del joven y optimice tanto su inicio como su mantenimiento en dicho estilo de vida. La investigación se apoya en una triangulación metodológica con técnicas cuantitativas, como el análisis descriptivo de los datos recogidos a través de un cuestionario y cualitativas, como el análisis de contenido de cuatro grupos de discusión. El escaso interés se establece como la principal barrera para iniciarse o mantenerse en la práctica físico-deportiva tanto en los estudiantes de Educación Secundaria Obligatoria (ESO) como en los universitarios. Ante esto se proponen determinadas claves educativas, a tener en cuenta desde edades tempranas, para aumentar la motivación en la población, en general, y en los jóvenes físicamente inactivos, en particular.

Palabras clave: Motivación, actividad físico-deportiva, ocio y educación.

Education key to increase the motivation of physically inactive young people. This study aims to determine, first, what are the reasons that lead young people to abandon physical and sport practice and, secondly, to discover why some young people never started in this type of experience, in order to establish an educational philosophy that involves the physical and sport practice of young people and that they optimize both their beginning and their maintenance in this lifestyle. This research is based on methodological triangulation with quantitative techniques such as descriptive analysis of data collected through a questionnaire and qualitative techniques, such as content analysis of four focus groups. The lack of interest is set as the main barrier to start or stay in physical and sports practice both in students of secondary school and in college students. Therefore, certain education key are proposals to be considered at an early age, to increase motivation among the population, in general, and physically inactive youth in particular.

Key words: Motivation, physical-sport activity, leisure and education.

Correspondencia: Eva Sanz Arazuri. Universidad de La Rioja. C/ Luis de Ulloa, s/n. 26004. Logroño. E-mail: eva.sanz@unirioja.es 
El estudio de la motivación se constituye en un aspecto relevante en el análisis de la práctica físico-deportiva, dado que nos ayuda a demarcar las causas del inicio o no inicio, así como las referentes al abandono o la continuidad en la misma.

Diferentes autores (Morilla, 1994; Reeve, 1994; Valdés, 1996) explican la motivación como una posible causa de nuestra conducta que se pone de manifiesto en la intensidad (cantidad de empeño empleado), dirección (atracción por ciertas situaciones) y persistencia (duración del sujeto en la actividad) hacia dicho comportamiento, quedando determinada por necesidades biológicas, cognitivas, afectivas y sociales.

Deci y Ryan (1985) sitúan este concepto en un plano multidimensional, integrado por la motivación intrínseca, la motivación extrínseca y, por último, la desmotivación. La primera se refiere a un compromiso voluntario hacia la actividad, fruto de la satisfacción personal y en ausencia de recompensas materiales y obligaciones externas. A su vez, la motivación extrínseca es la que procede del exterior, generalmente suscitada por recompensas materiales o sociales. Por último, la desmotivación, también denominada amotivación (Biddle, 1999) quedaría definida como el estado experimentado cuando el sujeto tiene poca o nula motivación para pretender un comportamiento. Apuntan los autores que un individuo desmotivado suele abrigar sentimientos de incompetencia, frustración, miedo, depresión y descontrol.

En síntesis, de acuerdo con Moreno, Cervelló y González-Cutre (2007), la motivación es un elemento clave para lograr el inicio y mantenimiento en la práctica físico-deportiva por constituirse en uno de los determinantes cardinales de nuestro comportamiento (Iso-Ahola y St.Clair, 2000), ya que lo estimula, lo gobierna y lo regula (Murray, 1964; Roberts, 2001), estableciéndose en un dispositivo psicológico que administra la dirección, la intensidad y la persistencia de la conducta (Kanfer, 1994; Sage, 1977).

En virtud a lo apuntado se plantea como objeto del presente estudio averiguar los motivos principales por los que los jóvenes rechazan la práctica físico-deportiva, cuestión que da lugar a su abandono o al hecho de no haber practicado nunca este tipo de actividades, así como indagar en los factores de desmotivación que se asocian tanto a la renuncia como a no iniciarse en dicha práctica.

En una primera aproximación, en numerosas investigaciones con jóvenes de Educación Secundaria Obligatoria (Arribas, 2005; Palou, Ponseti, Gili, Borrás y Vidal, 2005; Rodríguez-Allen, 2000), se descubre que el escaso interés hacia la práctica físicodeportiva provocado por el hecho de no sentir gusto por la actividad, el deseo de realizar otras prácticas, así como experimentar aburrimiento, se constituye en motivo destacado para no iniciar o abandonar la práctica físico-deportiva. En este caso, la fuente de desmotivación la encontramos en necesidades personales que condicionan desfavorablemente la motivación intrínseca hacia la práctica físico-deportiva.

Por otro lado, en poblaciones universitarias, se descubre que el escaso interés, el cansancio, el hecho de no contar con amigos para realizar la actividad, así como la pereza y la desgana, también son destacados como factores de desmotivación con origen en necesidades personales y sociales (Hernández-Rodríguez, 2001; López-Yeste, 1999; Ruiz-Juan, 2001; Sanz, 2005). Además, se revela que la falta de tiempo, suscitada en la 
mayoría de las ocasiones por las obligaciones académicas, se constituye en factor principal de desmotivación que desemboca en la no realización de actividad físicodeportiva (Arribas, 2005; Gálvez, 2004; Palou et al., 2005; Rodríguez-Allen, 2000).

Igualmente, la falta de tiempo, la incompatibilidad de horarios, así como las obligaciones laborales y educativas se constituyen en factores desmotivadores relevantes en poblaciones universitarias (Gómez, 2005; Sanz, 2005).

Por su parte, Gálvez (2004) añade que a los motivos temporales, como razones desmotivadoras, le sigue el relacionado con la ausencia de instalaciones accesibles. En esta línea, también Gómez (2005) y Sanz (2005) destacan la gran distancia a la que se encuentra la instalación deportiva como causa cardinal de no inicio o abandono de práctica.

En este sentido, la motivación intrínseca quedaría determinada por condicionantes externos relacionados con las cuestiones espacio-temporales, los cuales darían lugar a que la práctica quedase supeditada a otras actividades.

Otros autores (Lorenzo y Martín-Ayllón, 2005; Robbins, Pender y Kazanis, 2003) en sendos estudios con chicas adolescentes, demostraron que uno de los mayores obstáculos encontrados que se constituía en fuente de desmotivación para no practicar guardaba relación con una falta de seguridad en sí mismas o la ausencia de experiencias placenteras anteriores adaptadas a los intereses personales. En este caso, la motivación intrínseca quedaría condicionada, fundamentalmente, por factores personales, afectivos y emocionales.

Otros motivos, que presentan menor relevancia, son el sentimiento de incompetencia (Gálvez, 2004; Valdemoros, 2010), la disolución del equipo por causas ajenas, así como las desavenencias con el entrenador (Arribas, 2005), o los impedimentos de los padres para que sus hijos realicen la práctica (Nuviala, Ruiz-Juan y García-Montes, 2003; Valdemoros, Ponce de León, Ramos y Sanz, 2011).

Como apuntamos con anterioridad, nuestra pretensión se orienta a averiguar los motivos destacados por los que los jóvenes no inician o abandonan la práctica físicodeportiva, con el fin de establecer una filosofía educativa que envuelva este tipo de prácticas en el joven y optimice tanto su inicio como su mantenimiento $\mathrm{y}$, como consecuencia, favorecer su desarrollo integral dado que, como constatan Goñi e Infante (2010), se establece una relación positiva entre la práctica de actividad físico-deportiva y la satisfacción con la vida.

\section{METODO}

\section{Participantes}

La presente investigación se implementó con dos poblaciones juveniles diferentes: la universitaria (7.965 sujetos) y la de Educación Secundaria Obligatoria (11.259 escolares).

Las muestras quedaron configuradas por 1.340 sujetos universitarios y 1.978 estudiantes de Educación Secundaria Obligatoria para un nivel de confianza del 95\% y un error de muestreo de $+/-2$ sigmas. 
En los universitarios el método de muestreo utilizado fue por cuotas y proporcional atendiendo a la titulación y al sexo, mientras que en los jóvenes de Educación Secundaria Obligatoria el muestreo fue estratificado por conglomerados con afijación de las unidades de primera etapa de forma proporcional atendiendo al lugar de estudios (capital/provincia) y al tipo de centro (público/privado-concertado).

\section{Instrumentos}

El procedimiento utilizado estuvo basado en una triangulación metodológica con técnicas cuantitativas, como el análisis descriptivo de los datos recogidos a través de un cuestionario y cualitativas, como el análisis de contenido de unos grupos de discusión.

Para la técnica cuantitativa se utilizó una parte del cuestionario MACOFYD (Ponce de León, Sanz, Ramos y Valdemoros, 2010), ya validado y configurado por un total de 39 ítems, de los cuales se extrajeron los relativos a los motivos por los que los jóvenes nunca se han iniciado en la práctica físico-deportiva o la han abandonado.

El análisis y tratamiento de los datos recogidos con el cuestionario se realizó con la ayuda del programa estadístico SPSS 15.0, efectuando un análisis descriptivo univariado a partir de la distribución de frecuencias.

Respecto a los grupos de discusión la selección de los participantes fue estructural eligiendo los distintos colectivos según criterios de pertenencia, se tuvieron en cuenta los principios de homogeneidad -en cuanto colectivo educativo-, heterogeneidad- en función del sexo- y número. En el caso de la población universitaria se configuraron tres grupos de discusión para distintos grupos focales -uno del Personal Docente e Investigador (7 sujetos), otro del Personal de Administración y Servicios (6 sujetos) y otro del alumnado (8 estudiantes)-. En el caso de la población de ESO se constituyeron cuatro grupos de discusión con los agentes educativos más cercanos a los estudiantes (10 profesores de Educación Física, 8 profesores de otras áreas, 8 padresmadres y 15 estudiantes).

Se confeccionó un protocolo de seguimiento, cuyas cuestiones se recogen en Sanz (2005) y no declaraban específicamente el ámbito de los motivos objeto de estudio con el fin de obtener una información producto más de la espontaneidad que de declaraciones condicionadas por una dirección excesivamente marcada del tema de análisis.

Los relatos surgidos en estos grupos de discusión fueron tratados con el paquete informático $N U D^{*} I S T$, recogiéndose las unidades textuales referentes a las influencias internas y externas que podían afectar a la motivación en las experiencias de ocio físico-deportivo adolescente.

\section{Procedimiento}

Este estudio gira en torno a dos cuestiones, por una parte, conocer cuáles son las razones que llevan a los jóvenes a abandonar la práctica físico-deportiva y, por otra, detectar por qué algunos jóvenes nunca se iniciaron en este tipo de experiencias. 
Para profundizar en los motivos de abandono se establecieron 11 variables, mientras que para ahondar en los que les llevaron a no haber practicado nunca se determinaron 7 variables.

Tabla 1. Motivos de rechazo físico-deportivo

\begin{tabular}{|c|c|c|}
\hline $\begin{array}{l}\text { Motivos de } \\
\text { abandono }\end{array}$ & DEFINICIÓN DE VARIABLES & $\begin{array}{l}\text { Motivos de no haber } \\
\text { practicado nunca }\end{array}$ \\
\hline Escaso interés & $\begin{array}{l}\text { Pereza, acabar cansado de la jornada académica, } \\
\text { pérdida de atractivo por la rutina, por tener otras } \\
\text { preferencias de tiempo libre. }\end{array}$ & Escaso interés \\
\hline Temporales & $\begin{array}{l}\text { Falta de tiempo, por los estudios, horarios } \\
\text { incompatibles con las obligaciones cotidianas. }\end{array}$ & Temporales \\
\hline $\begin{array}{l}\text { Relacionados con la } \\
\text { oferta }\end{array}$ & $\begin{array}{l}\text { Ausencia de oferta, porque resulta caro, están lejos } \\
\text { las instalaciones, no hay instalaciones, no se } \\
\text { ofertan las actividades que interesan, saturación de } \\
\text { instalaciones. }\end{array}$ & Relacionados con la oferta \\
\hline Sociales & $\begin{array}{l}\text { No tengo con quién practicar, mis amigos no hacen, } \\
\text { falta de apoyo y estímulo. }\end{array}$ & Sociales \\
\hline Por salud & Prescripción médica, lesiones. & Por salud \\
\hline Familiares & Padres con otras preferencias. & Familiares \\
\hline Materiales & $\begin{array}{l}\text { Falta de medios materiales, pérdida o rotura de los } \\
\text { mismos. }\end{array}$ & \\
\hline Emocionales & $\begin{array}{l}\text { Por timidez, complejos, baja autoestima, no } \\
\text { sentirse a gusto realizando la actividad. }\end{array}$ & \\
\hline Por hábito & $\begin{array}{l}\text { Falta de hábito físico-deportivo, cambio de lugar, } \\
\text { zona o país, aparición de hábitos nocivos. }\end{array}$ & \\
\hline $\begin{array}{l}\text { Relacionados con el } \\
\text { ego }\end{array}$ & $\begin{array}{l}\text { Pocas competiciones, el equipo no tenía un nivel } \\
\text { bueno. }\end{array}$ & \\
\hline \multirow[t]{2}{*}{ Personales } & No se esgrime el motivo. & \\
\hline & $\begin{array}{l}\text { Falta de formación físico-deportiva, escasa cultura } \\
\text { física. }\end{array}$ & Por falta de preparación \\
\hline
\end{tabular}

\section{RESULTADOS}

Los datos obtenidos de este estudio señalan el escaso interés por la práctica físico-deportiva como la principal barrera que dificulta tanto el acceso como el mantenimiento de las experiencias físico-deportivas en el espacio de ocio, tanto para los estudiantes de Educación Secundaria Obligatoria como para los universitarios.

Esta afirmación se sustenta al revelarse el escaso interés por la práctica físicodeportiva como el motivo más señalado por los jóvenes para abandonar su práctica, en un $42.3 \%$ de los casos. Escaso interés en donde se hallan aspectos tales como la pereza, el hecho de acabar cansado de la jornada académica, la pérdida de atractivo por la rutina $o$, incluso, por tener otras preferencias de tiempo libre.

Del mismo modo, entre los universitarios riojanos, 4 de cada 10 expracticantes señalan el bajo interés como una de las razones que les llevaron a desatender estas vivencias motrices en su espacio de ocio.

Este escaso interés por la práctica físico-deportiva se constituye como una barrera mucho más sólida entre los que nunca se han ejercitado físicamente en su tiempo libre, ya que un $77 \%$ de los estudiantes de Educación Secundaria Obligatoria y un 70.8\% 
de estos universitarios declaran rechazar este tipo de prácticas por el escaso interés que les despiertan.

En consonancia con los resultados obtenidos en el análisis descriptivo, las percepciones mostradas por los distintos colectivos, en los grupos de discusión, concuerdan en que el escaso interés es uno de los motivos más importantes de abandono o de no haber practicado nunca. Punto de vista que se manifiesta en las siguientes expresiones:

Llegados a una edad, algunos se empiezan a cansar un poco. (Colectivo de alumnos).

A mí, en vez de castigarme sin hacer deporte, me castigan a hacer deporte. (Colectivo de alumnos).

Pienso que ahora, a esas edades concretamente (adolescencia), no tienen demasiado interés tampoco. (Colectivo de profesores de otras áreas).

Figura 1. Motivos de abandono de los estudiantes de Educación Secundaria Obligatoria

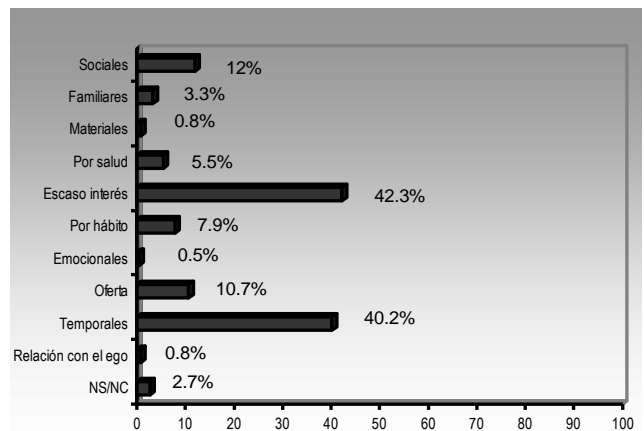

Figura 3. Motivos que llevan a no haber practicado nunca a los estudiantes de Educación Secundaria Obligatoria

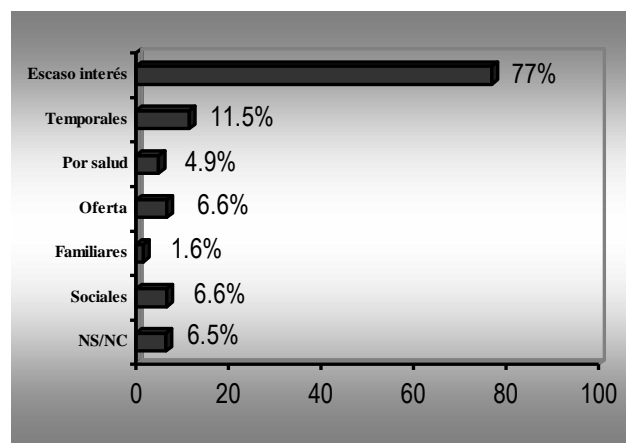

Figura 2. Motivos de abandono de los universitarios

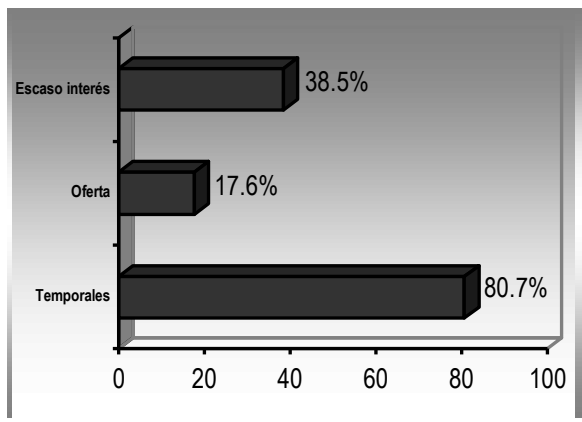

Figura 4. Motivos que llevan a no haber practicado nunca a los universitarios

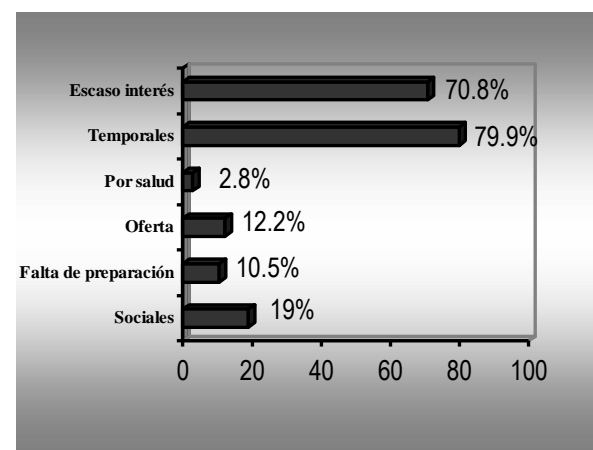


Las razones temporales, entre las que se incluyen la falta de tiempo, los estudios o los horarios incompatibles con las obligaciones cotidianas, constituyen una segunda barrera a tener en cuenta como obstáculo para acceder o mantener las experiencias físico-deportivas en el espacio de ocio. No obstante, estas cuestiones temporales afectan fundamentalmente a los universitarios, pero no tanto a los estudiantes de Secundaria Obligatoria, y entre estos últimos supone una mayor dificultad para continuar practicando que para acceder a este tipo de actividades.

Tal es así, que un $80.7 \%$ de universitarios que han abandonado sus vivencias físico-deportivas indican haberlo hecho por motivos temporales. En esta misma línea, un $79.9 \%$ de los universitarios que nunca han practicado señalan estos mismos motivos como causantes de su inactividad física.

Sin embargo, mientras estas razones son esgrimidas por un $40.2 \%$ de los escolares de Educación Secundaria Obligatoria que abandonaron su práctica, tan sólo un $11.5 \%$ de los que nunca han practicado dicen no haberlo hecho por cuestiones temporales.

Las elocuciones de los grupos de discusión, también nos señalan que los distintos colectivos perciben la falta de tiempo como otro de los motivos de abandono o de no haber practicado nunca, en especial los padres, tal y como se descubre con expresiones como:

Llega un momento que ya no hay tiempo para hacer de todo. (Colectivo de alumnos).

Yo creo que necesitan mucho tiempo para dedicar a los estudios, tanto en Secundaria como después en el Bachillerato, y el estar en un equipo implica mucho tiempo. (Colectivo de padres).

\section{DISCUSION}

El escaso interés hacia la práctica físico-deportiva es la principal barrera de acceso y mantenimiento de la misma, tanto de los estudiantes de Educación Secundaria Obligatoria como de los universitarios. Estos mismos resultados han sido obtenidos para otras muchas poblaciones de estudio, entre las que destacamos las analizadas por Hernández-Rodríguez (2001), López-Yeste (1999), Ruiz-Juan (2001) y Sanz (2005).

En este caso se trata de un factor interno, lo que dificulta la posibilidad de intervenir directamente sobre él; para hacerlo se debería profundizar en este aspecto intentando resolver una serie de cuestiones como: ¿por qué tienen bajo interés en las actividades físico-deportivas?, los que han abandonado ¿estaban más interesados cuando se iniciaron en la práctica?, ¿qué les ha hecho perder el interés?

Estos resultados ratifican la necesidad de que la intervención se centre en factores educativos y de concienciación hacia un ocio físico-deportivo.

Los especialistas físico-deportivos en el espacio de ocio deben intentar consolidar el hábito físico-deportivo y ayudar, a través de nuevos programas de intervención, a que estas prácticas se perciban como experiencias libres, satisfactorias y autotélicas. Basándonos en los objetivos de la pedagogía del ocio planteados por Cuenca 
(2004), se requiere una intervención educativa que potencie la práctica motriz de calidad y se centre fundamentalmente en la sensibilización, conocimiento y orientación de las vivencias físico-deportivas en el espacio de ocio.

Se hace necesario, pues, establecer unas claves educativas, a tener en cuenta desde edades tempranas, que pretendan, entre otras cosas: deportiva de ocio.

$\checkmark$ Concienciar sobre la importancia y beneficios de la práctica físico-

$\checkmark$ Fomentar el interés por este tipo de actividades de ocio físico-deportivo.

$\checkmark$ Dar a conocer la gran variedad de actividades y posibilidades de ocio físico-deportivo que le ofrece su entorno, así como aquellas que él mismo puede organizar y gestionar.

ocio.

$\checkmark$ Estimular el deseo y la participación en actividades físico-deportivas de

$\checkmark$ Orientar y generar la capacidad de elegir vivencias físico-deportivas en función de los deseos, motivaciones, expectativas y necesidades personales.

Sobre los condicionantes temporales como barrera importante de acceso y mantenimiento de la práctica físico-deportiva, fundamentalmente constatados en los universitarios, debemos tener en cuenta que un porcentaje considerable señala, más que la falta de tiempo, la incompatibilidad entre los horarios de la oferta y los de sus obligaciones educativas o laborales, lo que parece indicar que una oferta que atienda a los horarios libres de obligaciones de esta población podría frenar el abandono e incluso aumentar la actividad motriz.

Se considera necesario que futuras investigaciones contemplen la variable distribución del tiempo libre a lo largo de la jornada diaria, con el fin de dar respuesta a este desajuste.

\section{Agradecimientos}

Consejería de Educación, Cultura y Deporte del Gobierno de La Rioja el soporte financiero concedido al estudio dentro de los Proyectos "Fomenta" del Plan Riojano de I+D+I en el año 2007 (Ref. 2007/02).

\section{REFERENCIAS}

Arribas, S. (2005). La práctica de la actividad física y el deporte (PAFYD) en escolares de 15-18 años de Guipúzcoa: creencias sobre su utilidad y relación con la orientación motivacional, diversión y satisfacción. Bilbao: Servicio Editorial de la Universidad del País Vasco.

Biddle, S.J.H. (1999). Motivation and Perceptions of Control: Tracing its development and plotting its future in exercise and sport psychology. Journal of Sport and Exercise Psychology, 21, 1-23.

Cuenca, M. (2004). Pedagogía del Ocio: Modelos y Propuestas. Bilbao: Universidad de Deusto.

Deci, E.L. y Ryan, R.M. (1985). Intrinsic motivation and self-determination in human behaviour. New York: Plenum. 
Gálvez, A. (2004). Actividad física habitual de los adolescentes de la región de Murcia. Análisis de los motivos de práctica y abandono de la actividad físico-deportiva. Tesis doctoral. Facultad de Educación. Universidad de Murcia. Murcia. España.

Gómez-López, M. (2005). La actividad físico-deportiva en los centros almerienses de Educación Secundaria Post Obligatoria y en la Universidad de Almería. Evolución de los hábitos físico-deportivos de su alumnado. Tesis Doctoral. Facultad de Educación. Universidad de Almería. Almería, España.

Goñi, E. e Infante, G. (2010). Actividad físico-deportiva, autoconcepto físico y satisfacción con la vida. European Journal of Education and Psychology, 3(2), 199-208.

Hernández-Rodríguez, A.I. (2001). Análisis de la demanda de la comunidad universitaria almeriense en actividades físico-deportivas. Estudio de adecuación de la oferta. Almería: Servicio de publicaciones de la Universidad de Almería.

Iso-Ahola, S.E. y St.Clair, B. (2000). Toward a theory of exercise motivation. Quest, 52, 131-147.

Kanfer, R. (1994). Motivation. En N. Nicholson (Ed.), The Blackwell dictionary of organizational behaviour. Oxford: Blackwell publishers.

López-Yeste, A. (1999). El deporte de la Universidad Politécnica de Valencia, un estudio desde la psicología social del consumidor. Tesis Doctoral. Facultad de Ciencias Sociales. Universidad de Valencia. Valencia. España.

Lorenzo, F. y Martín Ayllón, F. (2005). Un estudio sobre intereses, actitudes y práctica de actividad físico-deportiva y género con la población de Educación Secundaria Obligatoria. Habilidad Motriz, 23-24, 5-19.

Moreno, J.A., Cervelló, E. y González-Cutre, D. (2007). Analizando la motivación en el deporte: un estudio a través de la teoría de la autodeterminación. Apuntes de Psicología, 1, 35-51

Morilla, M. (1994). El papel del entrenador en la motivación deportiva. El Entrenador Español de Fútbol, 60, 26-31.

Murray, E. (1964). Motivation and emotion. Englewood Cliffs, NJ: Prentice-Hall.

Nuviala, A., Ruiz-Juan, F. y García-Montes, M.E. (2003). Tiempo libre, ocio y actividad física en los adolescentes. La influencia de los padres. Retos. Nuevas tendencias en Educación Física, Deporte y Recreación, 6, 13-20.

Palou, P., Ponseti, F., Gili, M., Borrás, P. y Vidal, J. (2005). Motivos para el inicio, mantenimiento y abandono de la práctica deportiva de los preadolescentes de la isla de Mallorca. Apunts: Educación Física y Deportes, 81, 5-11.

Ponce de León, A., Sanz, E., Ramos, R. y Valdemoros, M.A. (2010). Cuestionario de motivaciones, actitudes y comportamientos en el ocio físico-deportivo juvenil. MACOFYD. Logroño: Universidad de La Rioja.

Reeve, J. (1994). Motivación y emoción. Madrid: McGraw-Hill.

Robbins, L.B., Pender, N.J. y Kazanis, A.S. (2003). Barriers to physical activity perceived by adolescent girls. Journal of Midwifery and Women's Health, 48, 206-212.

Roberts, G.C. (2001). Understanding the dynamics of motivation in physical activity: The influence of achievement goals on motivation processes. En G.C. Roberts (Ed.), Advances in motivation in sport and exercise (pp. 1-50). Champaign, IL: Human Kinetics.

Rodríguez-Allen, A. (2000). Adolescencia y Deporte. Oviedo: Ediciones Nobel.

Ruiz-Juan, F. (2001). Análisis diferencial de los comportamientos, motivaciones y demanda de actividades físico-deportivas del alumnado almeriense de enseñanza secundaria postobligatoria y de la Universidad de Almería. Almería: Servicio de publicaciones de la Universidad de Almería.

Sage, G. (1977). Introduction to motor behaviour: A neuropsychological approach. Reading, MA: Addison-Wesley. 
Sanz, E. (2005). La práctica físico-deportiva de tiempo libre en universitarios. Logroño: Universidad de La Rioja.

Suárez, F.A. (2005). El grupo de discusión. Una herramienta para la investigación cualitativa. Barcelona: Alertes.

Valdemoros, M.A. (2010). Los valores en el ocio físico-deportivo. Análisis y propuestas educativas. Logroño: Universidad de La Rioja.

Valdemoros, M.A., Ponce de León, A., Ramos, R. y Sanz, E. (2010). Pedagogía de la convivencia y educación no formal. Un estudio desde el ocio físico-deportivo, los valores y la familia. European Journal of Education and Psychology, 4(1), 33-49.

Valdés, H.M. (1996). La preparación psicológica del deportista. Mente y rendimiento humano. España: INDE publicaciones.

Recibido: 1 de marzo de 2010

Recepción Modificaciones: 1 de abril de 2010

Aceptado: 3 de mayo de 2010 